\title{
LA PRÁCTICA DEPORTIVA EN LA CONSTRUCCIÓN DE CIUDAD Y CIUDADANÍAS
}

SPORTS PRACTICE IN THE CONSTRUCTION OF CITY AND CITIZENSHIPS

Víctor Hugo Durán Camelo ${ }^{1}$

\section{Resumen}

\begin{abstract}
El presente escrito expone la reflexión del espacio académico Deporte y Ciudad en el marco de la Licenciatura en Deporte en la Universidad Pedagógica Nacional, sobre la multiplicidad de manifestaciones y correspondencias entre el desarrollo de las prácticas deportivas en la ciudad y la construcción de ciudad, subjetividades y formas de ciudadanía. Se hace evidente un campo de investigación multidisciplinar que observa las formas de uso del espacio y construcción de lugares de relación intersubjetiva, que transitan entre la institucionalidad de las prácticas y la anomia de las manifestaciones cotidianas del ciudadano. Las metodologías utilizadas circulan entre representaciones sociales, cartografías sociales y la comprensión de la ciudad acorde con la perspectiva de "ciudad educadora" (Declaración de Barcelona, 1990) y "aprendizaje situado" (Díaz, 2003), asignando un valor pedagógico a las prácticas deportivas que se desarrollan en el contexto de la ciudad, construyendo realidades y significados particulares y colectivos. Por otra parte, se resalta el deporte como hecho social y práctica cultural que integra dinámicas comunitarias, políticas, económicas y educativas (País, 2008), mostrando una radical transformación en la manera de asumir y desarrollar las prácticas deportivas, de habitar la ciudad y generar relaciones entre individuo, sociedad y cultura, cuestionadas a la luz de la política pública en salud, las manifestaciones juveniles, resistencias ciudadanas y la comprensión de los nuevos usos del espacio de la ciudad.
\end{abstract}

Palabras clave: Prácticas deportivas, ciudad, ciudadanía, subjetividad.

\section{Abstract}

This written reflection exposes sport academic space and city as part of the degree in sport at the National Pedagogical University, about the multiplicity of forms and correspondences between the development of sports activities in the city and city construction, subjectivities and forms of citizenship. A multidisciplinary field of research that looks at ways to use the space and build places of inter relationship opinion, transiting between institutional practices and anomie of daily protests of citizens is evident. The methodologies used are moved between social representations, social mapping and understanding of the city according to the perspective of Educating Cities (Barcelona Declaration, 1990) Situated Learning (Diaz, 2003) assigning a pedagogical value to sports activities taking place in the context of the city, construction individual and collective realities and meanings. Moreover, sport is highlighted as a social and cultural practice that integrates community dynamics, political, economic and educational (Country, 2008), showing a radical transformation in the way of taking and developing sports practices, inhabit the city and construct relationships between the individual, society and culture, questioned in light of public health policy, youth demonstrations, civic resistance and understanding of the new uses of city space.

Key words: Sporting Practice, City, Citizenship, Subjectivity.

Fecha de recepción: 15 de julio de 2014

Fecha de aprobación: 7 de noviembre de 2014

1 Doctorando en Educación, magíster en Educación, especialista en Pedagogía, Gerente de SISOE S.A.S. (Servicios Integrales en Salud Ocupacional y educación), docente catedrático Universidad Pedagógica Nacional, par académico Ministerio de Educación Nacional. Docente investigador cuya labor académica se ha perfilado hacia el campo de la interculturalidad, desarrollo comunitario, la lúdica, el deporte y, actualmente, la actividad física comunitaria y los procesos de formación virtual y a distancia. Miembro fundador de los grupos de investigación: SISOE SALUD, (GPAF) Gestión y Pedagogía de la Actividad Física y Conocimiento Pedagógico del Deporte, adscritos a Colciencias. Correo electrónico: guajiro78@gmail.com 
Para citar este artículo:

Durán, V.H. (2014). La práctica deportiva en la construcción de ciudad y ciudadanías. Revista Lúdica Pedagógica, (20), 27-35.

El presente escrito socializa el ejercicio de indagación desarrollado entre el año 2012 hasta el primer semestre del 2014, en el espacio académico denominado Deporte y Ciudad el cual hace parte de la Licenciatura en Deporte de la Universidad Pedagógica Nacional, haciendo evidente las relaciones múltiples que se establecen entre el deporte como categoría de análisis, sus prácticas y la comprensión de la ciudad como concepto (Hiernaux, 2006), pero fundamentalmente como construcción simbólica e intersubjetiva dependiente de las prácticas de los ciudadanos que la configuran.

En este sentido, se retoma el concepto de ciudad como construcción subjetiva y simbólica del espacio, significada de múltiples maneras por los sujetos y las relaciones que la componen, desplazando la idea de una ciudad determinada por sus límites geográficos o por su infraestructura física (Fukuda, 2010).

\section{SENTIDOS Y COMPRENSIONES DEL CONCEPTO DE CIUDAD}

Aunque la condición gregaria del hombre lo ha llevado a configurar formas diversas de agrupación, es evidente que la sociedad contemporánea ha encontrado y privilegiado la ciudad como la estructura humana que refleja de mejor manera un ideal de asociación, desarrollo y confluencia de prácticas.

La ciudad moderna se muestra como una estructura compleja de desarrollo social y como concepto polisémico que integra múltiples perspectivas de comprensión, entre ellas: una mirada de ciudad en oposición con el ámbito rural; como potencial humano atribuido al grado de desarrollo urbanístico, económico-industrial y cultural; y otras formas simbólicas de comprensión en relación con la apropiación (formas de habitar) y configuración del territorio, la identidad y proyectos particulares de ascenso social.

En este sentido, reflexionar sobre la ciudad y sus manifestaciones requiere mediaciones conceptuales o categorías de análisis que permitan su comprensión como fenómeno humano, y la identificación de imaginarios (Lindón, 2007), posibilidades y problemáticas, por lo cual se propuso en diferentes momentos de la indagación, el lugar, el ejercicio de la ciudadanía y las prácticas ciudadanas como ejes de análisis que permitieran rastrear dichas relaciones.
Los lugares de la ciudad

Según Páramo (2004), el lugar se define en la transformación que el sujeto hace del espacio físico-concreto, desde la atribución de características simbólicas que le permiten a los individuos construir sentidos y diversificar las formas de apropiación, de uso y arraigo sobre el espacio.

Desde la perspectiva de lugar surge una idea de ciudad comprendida a partir de la asignación de los límites reales o imaginarios, en este caso, para el ciudadano del común, la ciudad es ese espacio delimitado por un casco urbano organizado y diferenciado claramente de lo rural o el campo, como también es nombrado, que tiende a ser marginado y periférico. Asimismo, la ciudad como ámbito de desarrollo se configura en la impresión que poseen ciertas personas de cantidad, calidad y cualidad de la infraestructura urbanística: edificios, calles, escenarios disponibles, etc., así como servicios disponibles: salud, educación, acueducto y alcantarillado, recreación, entre otros. También cuenta en esa impresión la presencia de innovaciones culturales y tecnológicas, que son percibidas como obligatorias y necesarias en una sociedad moderna.

Por otra parte, la ciudad en cuanto diferenciación socio-cultural, es entendida como el conjunto de las diferencias individuales y colectivas en la producción simbólica de los sujetos sociales, lo cual incluye el nivel educativo y capital cultural de la población, es decir, la ciudad como lugar de la cultura.

Con respecto a las concepciones de los ciudadanos, se puede observar una marcada percepción positiva e idealizada de lo rural, como lugar que representa los valores humanos y la concepción tradicional de comunidad en términos de vínculos afectivos, actitud colaborativa y desinteresada, orden y convivencia pacífica; frente un imaginario de la ciudad como lugar que encarna los antivalores, la competencia, la individualidad, el desorden social, la violencia real y simbólica.

En este sentido, parece ser que la comprensión de la ciudad como lugar, implica indagar y reflexionar sobre la manera en que los ciudadanos apropian desde sus experiencias los espacios y las estructuras, la manera en que se configura el territorio y se establecen identidades particulares y colectivas sobre los lugares.

Diferentes autores han señalado que la ciudad moderna se ha transformado desde una perspectiva normativa y condicionante de los comportamientos sociales, con el propósito de hacer menos factible las apropiaciones o 
usos alternativos de sus espacios. Por ejemplo, Páramo (2004) señala la forma en que, en la idea del espacio público urbano, se han reducido drásticamente los sitios de encuentro y permanencia: bancas, plazoletas y aceras, postulando el centro comercial como la alternativa de la ciudad moderna para el encuentro con el otro.

En esta misma perspectiva, Tonucci (2009) afirma que los espacios de la ciudad se transforman progresivamente en sitios de paso apresurado hacia lugares de producción o consumo; pareciese que la ciudad moderna asiste hoy en día a una pérdida de sentido y relación de los sujetos con respecto a los lugares y la posibilidad de apropiación simbólica del territorio.

Sin embargo, según Berger y Luckmann (1986), se comprende una relación intersubjetiva que construye socialmente la realidad en la experiencia cotidiana y permite, más allá de las normas y características del espacio institucional, generar procesos de construcción del territorio que responden a características y condiciones contextuales y socioculturales de la realidad de los sujetos.

En consecuencia, el contexto, la realidad vivida y sus prácticas, son aspectos más relevantes en la configuración de referentes identitarios para los sujetos, ya que la idea de ciudad tiende a ser estructurada desde experiencias particulares y subjetivas. Desde otra perspectiva, el lugar de la ciudad es el lugar de la diferencia social, de los estratos socioeconómicos y el deseo de ascenso social, algunos autores previenen que la ciudad, en la medida en que estratifica sus espacios y particulariza la experiencia de los sujetos con base en lo socioeconómico (Uribe y Pardo, 2006), puede propiciar la discriminación y segregación de los individuos.

Esta comprensión nos ubica en una ciudad entendida como construcción subjetiva que hace posible una diversificación en la comprensión del espacio y su manifestación como construcción de lugar múltiple y diverso ligado a la experiencia de los ciudadanos. Dando cabida a perspectivas que la comprenden como lugar del encierro, guetos de discriminación socioeconómica (consciente o inconsciente), lugar de posibilidades de ascenso social como ideal ciudadano (Dammert, 2004), territorio de la experiencia y hogar que ancla los vínculos de ancestralidad.

\section{El ejercicio de la ciudadanía}

Podría pensarse que la condición de ciudadano se le asigna a toda persona que nace o se desarrolla en la vida de la ciudad; no obstante, a dicha denominación le asisten comportamientos, responsabilidades, derechos y deberes que configuran y fortalecen al individuo en el ejercicio de la ciudadanía. No basta con nacer o residir en la ciudad, la ciudadanía se adquiere en el reconocimiento de los derechos y deberes que le otorga la estructura social al ciudadano, condicionando su comportamiento y sentidos éticos en la relación con los otros, en términos de reconocimiento de la diversidad (género, edad, etnia, ideologías, religión, etc.), igualdad, equidad y alteridad, y participación pública y privada.

Por consiguiente, los ámbitos particulares y sus infraestructuras, los valores y tradiciones de la cultura y las condiciones socioeconómicas en que se encuentra inmerso el sujeto, se constituyen en aspectos que guían su pensamiento, comportamiento y ejercicio político, que determinan la forma de relacionarse en comunidad y genera contingencias colectivas (Páramo, 2010), que propician y fundamentan una identidad colectiva de ciudad y ciudadanía.

En consecuencia, la diversidad manifiesta en las relaciones y actuación del ciudadano daría cabida a una sociedad incluyente de aperturas y transformaciones, que permita la construcción de territorios, simbólicamente apropiados y enriquecidos por los sujetos y sus manifestaciones culturales, generando vínculos afectivos asociados al espacio cotidiano del individuo, susceptible de ser transformado posteriormente en territorio del ciudadano.

Por todo lo anterior, se concibe el ejercicio de la ciudadanía como un parámetro social, político y cultural en términos generales, que hace posible la manifestación de prácticas, creencias, comportamientos e ideologías basadas en la libertad, el reconocimiento de la diversidad y la igualdad de los ciudadanos.

\section{Las prácticas ciudadanas}

En esta propuesta se entiende la práctica ciudadana como esquemas simbólicos que definen el habitus de los individuos, transmitidos culturalmente con el fin de mantener vigentes procesos sociales en relación con la ciudadanía. Según Bourdieu, el habitus es:

Un mecanismo de representación, un sistema de esquemas perceptivos, evaluativos y pragmáticos que internaliza lo social en lo individual: Un sistema socialmente constituido de disposiciones estructuradas y estructurantes, adquirido mediante la Práctica, y siempre orientado hacia funciones prácticas. 
[...] Hablar de habitus es plantear que lo individual, e incluso lo Personal, lo subjetivo, es social, a saber, colectivo. El habitus es una subjetividad socializada. (Bourdieu, 1995, p. 87)

Cabe señalar que los contextos socioculturales determinan el tipo de prácticas individuales y las funciones que se le asignan en el colectivo social.

Por ello, la ciudad, como emblema de la forma de agrupación de la sociedad moderna, manifiesta una serie de prácticas que le son propias, concentrando intereses sobre ideales de cultura, salud, educación, bienestar, movilidad y relaciones interpersonales, entre otras.

A diferencia de zonas rurales, en donde las prácticas ciudadanas se enmarcan en lo tradicional del contexto, se replica algunas de las actividades propias de la ciudad, o son determinadas y restringidas por la posibilidad de acceso a bienes y servicios de la cultura.

En el caso de la ciudad, muchas de las prácticas se relacionan con la disposición espacial y el uso del espacio, por lo mismo, se complejizan en armonía con el grado de desarrollo de los escenarios y los servicios disponibles para los ciudadanos, podría decirse que son tan diversas y complejas como lo son las dinámicas de la ciudad. De igual manera, en la ciudad se pueden identificar prácticas formalmente institucionalizadas (como el caso del sistema educativo formal) y simultáneamente asistir a la aparición de un sinnúmero de ellas de manera informal, con capacidad para convocar diferentes colectivos sociales y generar identidades individuales y compartidas.

Un fenómeno particular es la presencia cada vez más marcada de las prácticas ciudadanas en espacios locales y comunitarios, como parques comunales, calles, callejones, lotes baldíos y espacios públicos en general, algunas de ellas a manera de resistencia juvenil frente a la pretensión de normalización de los comportamientos de los jóvenes o a la discriminación soterrada e invisibilizada que plantea la ciudad y su distinción a partir de estratos socioeconómicos.

En concordancia con lo anterior, Uribe y Pardo (2006) señalan que las ciudades se experimentan y viven de manera distinta según el estrato socioeconómico, esta barrera genera prácticas y mundos incomprensibles entre un sujeto y otro de la ciudad, muros infranqueables y desigualdades cada vez más marcadas que acentúan la mirada de desconfianza entre los distintos actores de estratos distintos.
La ciudad es la sumatoria de subjetividades presentes en los ciudadanos, la ciudad son sus prácticas, discursos y eventos cotidianos, la ciudad es en esencia la atribución simbólica que se hace a los espacios y lugares, a las infraestructuras, tiempos y relaciones de los sujetos.

Entonces, al señalar una práctica ciudadana particular como objeto de estudio (el deporte, por ejemplo), se está comprendiendo y configurando una forma particular de ciudad y de experiencia de la ciudad

\section{PROBLEMÁTICAS ACTUALES DE LA CIUDAD}

Desde el enfoque del déficit, Niemeyer (2006) muestra que son muchos los conflictos que le competen a la ciudad en términos de relaciones, lugares y estructuras, etc. Las principales problemáticas a tratar tienen que ver con la seguridad, reconocimiento del otro y la movilidad; las cuales han impulsado una serie de políticas públicas y transformaciones logísticas y socioculturales en búsqueda de una ciudad moderna y amable.

No obstante, las grandes ciudades deben enfrentar la percepción generalizada del ciudadano, que las determina como sitios caóticos e inseguros que deben ser contrarrestados a partir de prácticas de vigilancia, encierro y control político y judicial de las acciones de los individuos.

Por lo anterior, el conjunto residencial es la unidad de vivienda privilegiada en el nuevo modelo de ciudad, lugar que concentra los espacios y condiciones para satisfacer las necesidades de los ciudadanos, que van desde el parque y sus escenarios deportivos, hasta el supermercado, etc. Algunos Incluso, integran colegios y sitios de socialización como cafés o bares, evitando al máximo el hacer contacto con la ciudad del caos y la inseguridad.

En relación con lo anterior, la ciudad del encierro se constituye simultáneamente en la ciudad de la exclusión, puesto que las rejas, además de físicas, se tornan simbólicas y quienes se encuentran fuera son considerados diferentes y potencialmente peligrosos, desde criterios socioeconómicos, étnicos, ideológicos e incluso religiosos.

Por otra parte, se cuestiona si la infraestructura de la ciudad es adecuada o no frente a las necesidades de la población, pues se observa, en esta indagación particular, una problemática muy marcada en la disposición de vías y escenarios para la práctica deportiva, que tienden a vincularse con otras dinámicas sociales como la movilidad, consumo de psicoactivos, inseguridad y violencia. 
Del mismo modo, la carencia, el abandono o la inadecuada disposición de la infraestructura tienen la capacidad de alterar el comportamiento ciudadano. Es muy común hoy en día observar cómo algunos parques barriales abandonados han sido apropiados de otras maneras, constituyéndose en sitios de consumo, tráfico y delincuencia. De igual manera, calles poco iluminadas, obras inconclusas o malos diseños en términos de movilidad (puentes peatonales y ciclo rutas) se tornan un riesgo en la seguridad de los transeúntes.

Por lo mismo, la movilidad, como problemática de la ciudad, es reconocida a nivel mundial. Las ciudades se perciben como sitios caóticos repletos de automóviles, motocicletas y ciclomotores que disputan un precario espacio en las vías de la ciudad, sin mencionar el alto impacto ambiental que genera esta situación (Alguacil, 2000); lo que obliga a establecer en muchas ciudades del mundo, estrategias no muy bien recibidas por el grueso de la población, como pico y placa de automóviles, la peatonalización de ciertas zonas y peajes urbanos.

Ahora bien, las reflexiones sobre ciudad en el espacio académico Deporte y Ciudad se justifican en la medida en que se comprenda de qué manera una práctica ciudadana, como lo es el deporte o sus afines, puede asumirse en cuanto práctica mediadora significativa en la intervención de las diferentes problemáticas y percepciones de ciudad, anteriormente señaladas. Dado que aún esta relación no se encuentra determinada, los distintos procedimientos metodológicos de indagación propuestos en el espacio académico tienen la función de identificar y evidenciar estas correspondencias, hacerlas explicitas y colocarlas en función de los procesos pedagógicos que le competen misionalmente a la Universidad Pedagógica Nacional y al licenciado en Deporte de manera particular.

\section{METODOLOGÍAS DE INDAGACIÓN}

Las diferentes actividades de indagación alrededor de la temática "deporte y ciudad" se desarrollaron con el grupo de estudiantes del espacio académico entre el año 2012 y primer semestre del 2014, utilizando con este propósito una variedad de metodologías, técnicas e instrumentos aplicados a personas del común en ámbitos variados de la ciudad. Entre las metodologías más significativas aplicadas, se encuentran las representaciones sociales propuesta por Moscovici (1979), buscando establecer la relación entre las prácticas deportivas llevadas a cabo en diferentes lugares de la ciudad y las concepciones de los ciudadanos.

Asimismo, se incluyó la cartografía social (Diez et al., 2010) como una forma de comprender gráficamente las relaciones que se dan entre diferentes manifestaciones de las prácticas deportivas y el uso de los espacios de la ciudad, en cierta forma esta metodología se asume como una radiografía de las dinámicas sociales enfocadas en la práctica deportiva.

El aporte de las metodologías aplicadas al espacio académico hizo posible la diversificación del concepto de deporte y sus prácticas, al mapear la realidad se generan nuevas comprensiones sobre la manera en que la ciudadanía asume dicha práctica y actúa desde unos imaginarios socialmente construidos. Sin embargo, fue necesaria la inclusión de dos nuevas perspectivas teóricas para determinar la intencionalidad pedagógica de lo deportivo y su uso en los contextos particulares de la ciudad.

En primera instancia, la perspectiva de ciudad educadora (Villa y Moncada, 1998) en la exploración de su potencial para la formación del ciudadano en su experiencia cotidiana. Según Villa y Moncada (1998), se le atribuye a las dinámicas de la ciudad, su infraestructura, espacios y lugares, una función educadora por sí misma, desde la perspectiva del espacio académico, daría una idea de qué tanto el ciudadano adquiere un capital cultural en torno a la práctica del deporte y genera representaciones simbólicas, identidades y formas de creación de territorios.

En segunda instancia, fue incluida la perspectiva de aprendizaje situado (Kirk, Brooker y Braiuka, 2003), enmarcada en la intencionalidad pedagógica que vincula el aprendizaje a las características de contextos y sujetos, por lo cual se postula como intención pedagógica, que la estructura del sistema educativo debe formar en la práctica deportiva y afines (Herrera, 2007), atendiendo a características y necesidades contextuales de los sujetos.

Así, las perspectivas ciudad educadora y aprendizaje situado le aportan un valor pedagógico a las prácticas deportivas, lugares, espacios y dinámicas sociales que se desarrollan en el contexto de la ciudad y a la manifestación de subjetividades que se reinventan permanentemente desde la interacción de los sujetos en estos lugares.

Por último, las indagaciones contemplaron la aplicación de diferentes instrumentos, entre los cuales se resaltan: las entrevistas semiestructuradas, las encuestas de 
opción múltiple, escalas likert, y mapas colectivos; cada uno de ellos aportó de manera significativa al entendimiento de la relación deporte y ciudad.

\section{HALLAZGOS}

\section{Política pública y construcción de ciudadanías mediadas por la práctica deportiva}

Para la presente indagación se entiende la política pública como propósito y acción de un gobierno expresada en políticas y programas:

Tanto la política como las políticas públicas tienen que ver con el poder social. Pero, mientras la política es un concepto amplio, relativo al poder en general, las políticas públicas corresponden a soluciones específicas de cómo manejar los asuntos públicos o situaciones socialmente problemáticas. (Lahera, 2004, p. 7)

Por ello, el espíritu de la práctica deportiva contempla la exaltación de valores humanos y cualidades físicas superiores, que en el ámbito de lo competitivo se ponen a prueba en términos individuales y colectivos. Es así como el desarrollo de los juegos olímpicos lleva consigo un ideal implícito de ser humano, que permite reflexiones sobre el tipo de sujeto a construir socialmente para ser sustentado de una u otra manera (en el caso señalado) en las prácticas deportivas.

En este sentido, las principales críticas al deporte hacen evidente la exagerada intencionalidad competitiva (pese a que la Ley 181 reconoce manifestaciones de lo deportivo que desbordan esta pretensión); lo excluyente de las prácticas frente a la perspectiva de género, discapacidad (Neira, 2011), etnia y juventud; su enfoque exageradamente económico; y la relativa y precaria participación en la construcción social.

Como respuesta, se presentan las nuevas pretensiones de la política pública en deporte y salud, por ejemplo, el programa Bogotá más Activa 2009-2019, que en su formulación y accionar apunta a la construcción de sujetos sociales cuyos valores y habilidades desborden la demostración de condiciones atléticas, según el lema de los juegos olímpicos: Citius, Altius, Fortius; ubicando socialmente otros aspectos al ideal de ciudadano: más honesto, colaborativo, sensible, compasivo, amable, altruista, más humano, etc.

Por consiguiente, con la pretensión de constituir un ideal de ciudadano desde la política pública, se con- solida socialmente el deporte y sus prácticas institucionalizadas, a manera de cuerpo normativo, social, cultural; que representa ideas, valores, creencias, normas y formas de relación; llevando a la política pública en deporte a generar programas y proyectos tendentes a intervenir los comportamientos ciudadanos, generar nuevos imaginarios y relaciones con el espacio y con las intersubjetividades.

Las prácticas deportivas institucionalizadas asumen formas diversas de acuerdo con las manifestaciones establecidas por la Ley 181, algunas con mayor formalidad que otras, es el caso de los institutos de deportes, comités, federaciones, ligas y clubes deportivos, frente a otros informales: festivales barriales, agrupaciones comunitarias, encuentros casuales de socialización entre amigos y prácticas individuales con fines múltiples.

En relación con lo anterior, las indagaciones muestran un fuerte vínculo entre el desarrollo de las entidades deportivas, las políticas públicas estatales que promueven normatividades en términos de lo deportivo y el comportamiento del individuo, encausándolo, a manera de normalización de su comportamiento.

La normalización de las prácticas deportivas desde las instituciones sociales implica un propósito de contención de las manifestaciones humanas, polarizadas entre lo que se considera socialmente positivo y aquellas prácticas estigmatizadas en el ámbito social.

Así, el orden social se postula como un propósito fundamental de la política pública y, para ello, ciertas prácticas sociales se tornan hegemónicas, entre ellas las deportivas, sobre todo aquellas enfocadas hacia las manifestaciones juveniles, enmarcadas y reguladas por la disposición y forma de uso de los escenarios deportivos.

Por lo anterior, algunas prácticas deportivas son estigmatizadas o vinculadas con prácticas vandálicas, asociales o marginales y, en consecuencia, son restringidas normativamente, contenidas desde la intervención institucional o reguladas espacialmente a partir del surgimiento de infraestructuras dispuestas para su práctica adecuada, es decir, bajo un estructura prediseñada, reglamentada y espacialmente definida. Para analizar lo anterior, se puede usar el ejemplo de los jóvenes skaters, quienes por mucho tiempo han usado los espacios disponibles de la ciudad: aceras, bancas, escaleras, bolardos, entre otros, para el desarrollo de su práctica deportiva, debido a esto han sido estigmatizados por la autoridad local y la ciudadanía en general como "aquellos jóvenes que dañan el espacio público". 
Para contener el skate rollers, considerada práctica contraventora de la norma, desde la política pública, las instituciones estatales y deportivas optan por incluir en los megaparques o parques en concesión, la construcción de escenarios deportivos que de manera explícita se destinan para dicha práctica.

Parte de la comprensión de la ciudad como referente social se basa en su dinamismo, en el surgimiento de nuevas prácticas, representaciones y comportamientos sociales, etc. Muy por el contrario a la estigmatización percibida, esta producción del ciudadano debería verse como potencial social, educativo y cultural, medida desde la variedad de producciones simbólicas subjetivas y prácticas que se llevan a cabo en el ejercicio de la ciudadanía.

Como se muestra, la relación entre escenarios de la ciudad y prácticas deportivas son directas cuando se conciben como parte de una estrategia social de regulación del comportamiento de los individuos. No obstante, se debe tener presente que los ciudadanos y sus subjetividades transforman permanentemente estas manifestaciones, dando cabida a una forma dialéctica y dialógica de participación política, y generando la imperiosa necesidad de establecer acuerdos ciudadanos, entre los intereses deportivos (ya sean individuales o masificados) y el uso y apropiación de los espacios de la ciudad.

En la medida en que dichas prácticas sean reconocidas o no por la institucionalidad del Estado, se estaría haciendo referencia a una trasformación en la postura general de la ciudad y la ciudadanía frente a sus manifestaciones, desde un referente de equidad, inclusión y reconocimiento de la diversidad en este caso particular, con base en las prácticas deportivas.

\section{El deporte como práctica social y cultural}

Según lo planteado por la Ley 181 (1995), el deporte se define como "la específica conducta humana caracterizada por una actitud lúdica y de afán competitivo de comprobación o desafío, expresada mediante el ejercicio corporal y mental, dentro de disciplinas y normas preestablecidas orientadas a generar valores morales, cívicos y sociales" (título IV, cap. I, art. 15). Para el ciudadano común, el concepto de deporte se manifiesta como imaginarios alrededor del movimiento y la experiencia individual, dando cuenta de un amplio margen de posibilidades, entre ellas la recreación, actividad física, lúdica, juego y el ejercicio físico.

Lo anterior tiene implicaciones en las prácticas deportivas que el ciudadano propone para sí mismo, de igual manera afecta la forma de uso de los espacios de la ciudad, debido a que la calle o los senderos usados para trotar podrían ser catalogados —desde los imaginarios- como escenarios deportivos.

No obstante, al indagar por los lugares comunes culturalmente reconocidos para el desarrollo de la práctica deportiva, se pudo observar que independientemente de que se practique o no deporte, los megaparques son los sitios donde las personas del común ubican de manera principal dichas prácticas.

Por otra parte, se ubican los parques barriales, los gimnasios y la calle, en el caso de Bogotá, en cuanto que allí se desarrollan prácticas de fin de semana como la ciclovía y recreovía. La Unidad Deportiva El Salitre y las respectivas ligas deportivas son señaladas como lugares de práctica, como un caso particular, puesto que solo quienes han sido deportistas o han tenido alguna experiencia directa con el entrenamiento deportivo en estos lugares, ubican allí las prácticas deportivas.

Por último, se mencionaron otro tipo de escenarios pero su referencia no fue significativa en la indagación, entre ellos se mencionaron los sitios al aire libre como montañas y descensos rocosos, en el caso del montañismo y el ciclo montañismo.

En cuanto a la función que los ciudadanos le asignan a la práctica deportiva, se determinaron cuatro criterios generales: la salud; la socialización con familiares y amigos, en otras palabras, el deporte como un medio para el encuentro con el otro; la utilización del tiempo libre (Rodríguez \& Agulló, 1999); y, por último, la competencia, aunque esta pareciera ser la característica más reconocida y difundida en la práctica deportiva, en relación con las motivaciones de los ciudadanos no fue relevante.

\section{A MANERA DE CONCLUSIONES PARCIALES}

La ciudad y las manifestaciones del deporte ponen en evidencia que las prácticas tienden a ser muchas y diversas para cada ciudadano. Los jóvenes, los deportistas, los adultos trabajadores, las familias, el comercio, etc., cada actor comprende y difunde una idea de la práctica deportiva que conlleva a prácticas ciudadanas y representaciones del individuo.

Por su parte, la política pública centrada en el deporte, proyecta, incentiva y determina transformaciones del ciudadano, para acercarlo cada vez más a encarnar un ideal de hombre que reconoce en el movimiento humano un potencial para la consolidación de los valores deseables. 
Sin embargo, muchas de estas formas de construcción de ciudad y de ciudadanías se constituyen como formas de resistencia frente a la intencionalidad política de normativizar, normalizar, contener y encausar las producciones simbólicas de los ciudadanos, anclándose definitivamente en la actualización de la cultura.

Por lo tanto, se evidencia, desde el deporte en particular, que algunas prácticas culturales tienden a ser transgresoras del orden imperante y, por lo mismo, innovadoras, estas desbordan los conceptos y manifestaciones de las prácticas para dar origen a deportes llamados alternativos o no convencionales.

Se reconoce la ciudad como lugar de encuentros culturales, de grupos urbanos y subculturas, desde allí, las prácticas deportivas también se constituyen en un factor social de cambio (Puig, 1991) de resistencias, de innovación y actualización cultural, que permiten al deporte formal y alternativo encontrar sujetos y espacios para su desarrollo y generar identidades que sostienen y hacen que perduren dichas prácticas.

De alguna manera, las prácticas deportivas llevadas a cabo por individuos o pequeños grupos, por ejemplo, el utilizar las barras en los parques, descender colinas en bicicleta, superar obstáculos a pie, patín, monopatín o bicicleta, escalar obstáculos, etc. Son la base para que existan hoy en día una serie de prácticas deportivas reconocidas y apropiadas por una cantidad significativa de ciudadanos, muchos de ellos jóvenes que buscan alternativas frente a las prácticas tradicionales del deporte, dando origen al gimbarr (gimnasia en barras), parkour, derrapadas en triciclo, skate roller, BMX, estilo libre, escalada libre en edificio, entre otras prácticas deportivas.

Por último, se comprende desde este ejercicio de indagación que el deporte, en cuanto categoría, y la práctica deportiva como manifestación del individuo, conduce a la construcción de múltiples formas de comprender la ciudad anclándose desde la subjetividad, a las territorializaciones, representaciones simbólicas y construcción de identidad en los ciudadanos. De igual manera, la tensión entre la política pública, las prácticas institucionalizadas y las prácticas espontáneas que surgen inicialmente como resistencia frente a la intención de normalizar las manifestaciones del individuo, dan origen a formas diversas de ejercer la ciudadanía, mostrándose como una forma de ejercicio político del ciudadano.

\section{REFERENCIAS}

Alcaldía Mayor de Bogotá (2009). Bogotá más activa, Política pública de deporte, recreación y actividad física para Bogotá 2009 - 2019. Recuperado en http://www.culturarecreacionydeporte.gov.co/ sites/default/files/politica_publica_de_deporte_ recreacion_y_actividad_fisica_2009-2019.pdf

Alguacil, G. (2000). Calidad de vida y modelo de ciudad. Madrid: Instituto Juan De Herrera.

Berger, P. y Luckmann, T. (1986). La construcción social de la realidad. Buenos Aires: Editorial Amorrortu.

Bourdieu, P. (1995). Las reglas del arte: génesis y estructura del campo literario. Madrid: Editorial Anagrama.

Dammert, L. (2004). ¿Ciudad sin ciudadanos? fragmentación, segregación y temor en Santiago. Revista Eure, 30(091), 87-96.

Declaración de Barcelona (1990). Carta de ciudades educadoras I Congreso Internacional, Barcelona, España.

Díaz, B. (2003). Cognición situada y estrategias para el aprendizaje significativo. Revista electrónica de investigación educativa, 5(2), 2-13.

Diez, T., Escudero, H., Carballeda, A., Barberena, M., Hallak, Z., Rocha, E., y Romero, N. (2010). Cartografía social: investigaciones e intervención desde las ciencias sociales: métodos y experiencias de aplicación. Universitaria de la Patagonia. Recuperado de http:// www.margen.org/Libro1.pdf

Fukuda, H. (2010). Planeación urbana en Curitiba, México. Revista Quivera, 12(1), 52-69.

Herrera, C. X. (2007). De la escuela a la ciudad: cuerpos civilizados, sujetos modernos, el caso colombiano en la primera mitad del siglo XX. Medellín: Funámbulos editores.

Hiernaux, D. (2006). Repensar la ciudad: la dimensión ontológica de lo urbano. Revista Liminar. Estudios sociales y humanísticos, 4(2), 7-17.

Kirk, D., Brooker, R., y Braiuka, S. (2003). Enseñanza de los juegos para la comprensión: perspectiva situada en el aprendizaje de los estudiantes. Revista ágora para la EF y el deporte, 2(3), 154-164.

Lahera, P. (2004). Política y políticas públicas. Serie Políticas Sociales. Santiago de Chile: Naciones Unidas - CEPAL. 
Lindón, A. (2007). La ciudad y la vida urbana a través de los imaginarios urbanos. Revista Eure, 33(99), 7-16.

Moscovici, S. (1979). El psicoanálisis, su imagen y su público. Buenos Aires: Editorial Huemul S.A.

Neira, T. (2011). Determinantes sociales que promueven la inclusión/exclusión al deporte adaptado en el ámbito competitivo (tesis de maestría). Universidad Nacional de Colombia, Bogotá, Colombia.

Niemeyer, B. (2006). El aprendizaje situado: una oportunidad para escapar del enfoque del déficit. Revista de educación, 341, 99-121.

País, A. (2008). Construyendo refugios culturales. El caso del programa cultural en barrios. Revista austral de ciencias sociales, 14, 5-22.

Páramo, P. (2004). Algunos conceptos para una perspectiva optimista de vivir la ciudad. Revista Territorios, 10(11), 91-109.

Páramo. P. (2010). Aprendizaje situado: creación y modificación de prácticas sociales en el espacio público urbano. Revista Psicologia \& sociedade, 22(1), 130-138.
Puig, N. (1991). El deporte en la perspectiva del año 2000. Revista de Sociología, 38, 123-141.

Rodríguez, S., y Agulló, T. (1999). Estilos de vida, cultura, ocio y tiempo libre de los estudiantes universitarios. Revista Psicothema, 11(2), 247-259.

Tonucci, F. (2009). Ciudades a escala humana: la ciudad de los niños. Revista de educación, número extraordinario, 147-168.

Uribe, M., y Pardo, P. (2006). La ciudad vivida: movilidad espacial y representaciones sobre la estratificación social en Bogotá. Revista Universitas Humanística, 62, 169-203.

Vélez, T. Rátiva, G. y Varela, C. (2012). Cartografía social como metodología participativa y colaborativa de investigación en el territorio afrodescendiente de la cuenca alta del río cauca cuadernos de geografía. Revista colombiana de geografía, 21(2), 59-73.

Villa, M., y Moncada, C. (1998). Ciudad educadora estado del arte en Colombia. Recuperado de http:// www.region.org.co/index.php/es/publicamos/ libros/103-ciudad-educadora-estado-del-arte-en-colombia-1998. 